\title{
ESQUIZENCEFALIA
}

\section{Relato de onze casos}

\author{
João Guilherme Pires do Amaral1 , Ricardo Hideki Yanaga'2, Heloyse Jungblut Geissler'2, \\ Arnolfo de Carvalho Neto ${ }^{3}$, Isac Bruck ${ }^{4}$, Sergio A. Antoniuk ${ }^{4}$
}

\begin{abstract}
RESUMO - O presente estudo comparou achados de tomografia computadorizada (TC) com aspectos clínicos de pacientes com esquizencefalia. Sete crianças eram meninas e quatro meninos. Seis apresentavam tetraparesia, três hemiparesia e um não tinha déficit motor. Seis eram epilépticos. Dez apresentavam atraso do desenvolvimento neuropsicomotor. De acordo com os achados encontrados na TC, sete pacientes apresentaram fendas bilaterais e quatro defeito unilateral. Oito tinham fendas de lábios abertos e quatro de lábios fechados. A anomalia associada mais comum foi a ausência de septo pelúcido $(n=9)$, seguida por nódulos subependimários $(n=4)$, hidrocefalia $(n=2)$ e microcefalia $(n=1)$. Embora a ressonância magnética seja 0 exame padrão-ouro para diagnosticar anomalias de migração neuronal, a TC é mais disponível e pode ser útil no diagnóstico das esquizencefalias, se os aspectos típicos forem reconhecidos.
\end{abstract}

PALAVRAS-CHAVE: desordem de migração neuronal, esquizencefalia, aspectos clínicos, TC.

\section{Schizencephaly: report of eleven cases}

\begin{abstract}
A retrospective study comparing clinical and computerized tomography (CT) in 11 patients diagnosed as having schizencephaly was conducted. Seven of these patients were girls and four boys. Six of them had tetraparesis, three hemiparesis and one no motor deficits. Six had epilepsy and ten developmental delay. On CT examinations, 7 patients were found as having bilateral clefts and four unilateral defect. Eight had opened lip clefts and four had a closed lip defect. The commonest associated anomaly was an absent septum pellucidum $(n=9)$, followed by subependymal nodules $(n=4)$, hydrocephalus $(n=2)$ and microcephaly $(n=1)$. Despite magnetic resonance image is the gold-standard to diagnose neuronal migration anomalies, CT can be useful in showing typical aspects of schizencephaly.
\end{abstract}

KEY WORDS: neuronal migration disorder, schizencephaly, clinical aspects, CT.

A esquizencefalia caracteriza-se por fendas, que se estendem da superfície pial até a ependimária com as bordas revestidas por substância cinzenta. Classificam-se em dois tipos: o tipo I que se caracteriza pela presença de lábios fechados, ou seja, com as duas corticais justapostas; e o tipo II que apresenta lábios abertos, estando a fenda preenchida por líquido cefalorraquidiano. As fendas podem ser uni ou bilaterais, afetam com maior frequência as regiões peri-silvianas e estão, muitas vezes, associadas a outras malformações, especialmente defeitos de migração ${ }^{1-5}$.

As manifestações clínicas são variadas, mas as mais encontradas são o déficit motor, a epilepsia, e o retardo mental ${ }^{3,5,6}$. Alguns pacientes não apresentam alterações neurológicas, sendo a malformação apenas um achado de exame ${ }^{7}$. Os modernos métodos de imagem facilitaram em muito a identificação destas lesões, que antigamente eram consideradas raras.

O objetivo do presente estudo é correlacionar os achados de tomografia computadorizada (TC) com as manifestações clínicas dos pacientes do Hospital de Clínicas da Universidade Federal do Paraná (HCUFPR), que apresentavam esquizencefalia e comparálos com a literatura.

\section{MÉTODO}

Foi realizado estudo descritivo de 11 pacientes, através da análise retrospectiva de seus prontuários, que tiveram o diagnóstico de esquizencefalia nos exames de TC de crânio, realizados no Serviço de Radiologia do HC-UFPR.

Hospital de Clínicas da Universidade Federal do Paraná (UFPR), Curitiba, Paraná, Brasil: ${ }^{1}$ Residente de Radiologia; ${ }^{2}$ Médicos; ${ }^{3}$ Radiologista e Professor do Serviço de Radiologia; ${ }^{4}$ Neuropediatra e Professor do Departamento de Pediatria. 
Foram registrados sexo, idade dos pacientes na data do diagnóstico, antecedentes obstétricos, desenvolvimento neuropsicomotor (DNPM), déficits motores e história de crises convulsivas (idade de início e tipos de crise).

\section{RESULTADOS}

Os dados obtidos na revisão dos prontuários, bem como seus achados tomográficos, estão reunidos nas Tabelas 1 e 2.

Foi observada predominância do sexo feminino: 7 meninas para 4 meninos. Predominaram as fendas de lábios abertos $(n=8)$ e bilaterais $(n=7)$. A anomalia associada mais observada, foi a ausência de septo pelúcido $(n=9)$, associada ou não a nódulos subependimários $(n=4)$. O quadro clínico preponderante foi o de tetraparesia espástica (incluindo hipertonia generalizada) em 7 crianças. Hemiparesia ocorreu em 1 caso de fenda unilateral e bilateral, respectivamente. Somente um caso apresentou exame neurológico e DNPM normais (Paciente 6). Epilepsia esteve presente em 6 casos, sendo as crises generalizadas em 3 deles. Disfagia foi observada em 3 (Pacientes 2 , 3 e 11). Microcefalia foi evidenciada em uma criança (Paciente 1) e hidrocefalia estava associada em duas (Pacientes 6 e 9). Em 3 pacientes foram demonstradas calcificações puntiformes.

\section{DISCUSSÃO}

A esquizencefalia é malformação cerebral que foi descrita pela primeira vez em 1859 por $\mathrm{Haeschl}^{8}$. O termo esquizencefalia, porém, foi introduzido somente em 1946 por Yakovlev e Wadsworth, referindo-se a "uma fenda com persistência de espessa e rica camada de substância cinzenta na profundidade, de extensão do córtex até o ventrículo e com orientação simétrica bilateralmente" ${ }^{\prime 1,2,3,9}$. Ela é considerada uma anomalia da migração neuronal sendo que, neste grupo, incluem-se também a agiria, a polimicrogiria, a paquigiria e a heterotopia da substância cinzenta ${ }^{3,5,10} \mathrm{~A}$ etiologia mais aceita é a de uma falha segmentar na formação de porção da matriz germinativa ou na migração de neuroblastos primitivos, formando fendas cerebrais, que podem ser uni ou bilaterais ${ }^{1,6,11}$. A alta incidência de lesões bilaterais na região peri-silviana, tem sido usada para reforçar a idéia de que a hipotensão fetal e dano cortical isquêmico, reside nessa região por ser o maior território vascular do cérebro ${ }^{12}$. Todavia, insultos infecciosos e danos diretos de origem tóxica, não podem ser descartados dentre os fatores etiológicos ${ }^{13}$. Nos nossos casos, encontramos 3 pacientes com calcificações puntiformes, o que sugere a possibilidade de infecção intra-uterina como causa da mal formação. Baseado na idéia de que a esquizencefalia tem fisiopatologia semelhante às displasias corticais, é proposto que o insulto cerebral ocorra entre o $2 .^{\circ}$ e o $5 .^{\circ}$ mês de gestação ${ }^{13}$.

As fendas podem ser simétricas ou não e o córtex que a circunda é sempre anormal. As alterações podem variar de polimicrogiria ${ }^{14}$, que se estende por

Tabela 1. Dados referentes aos pacientes com diagnóstico de esquizencefalia e seus achados de imagem.

\begin{tabular}{lccccl}
\hline Pac & Idade (anos) & Sexo & & & Imagem \\
\cline { 3 - 5 } & & & Tipo da fenda & Simetria & Outros achados \\
\hline 1 & $2 \mathrm{a}$ & $\mathrm{M}$ & Lábios abertos & Bilateral & Ausência de septo pelúcido \\
2 & $3 \mathrm{a}$ & $\mathrm{M}$ & Lábios fechados & Unilateral & Ausência de septo pelúcido \\
3 & $10 \mathrm{a}$ & $\mathrm{F}$ & Lábios abertos & Unilateral & Ausência de septo pelúcido \\
4 & $1 \mathrm{a}$ & $\mathrm{F}$ & Lábios abertos & Bilateral & Ausência de septo pelúcido \\
5 & $11 \mathrm{a}$ & $\mathrm{M}$ & Lábios abertos & Unilateral & - \\
6 & $1 \mathrm{a}$ & $\mathrm{F}$ & Lábios abertos & Bilateral & Ausência de septo pelúcido \\
7 & $5 \mathrm{a}$ & $\mathrm{F}$ & Lábios abertos & Bilateral & Nódulos ependimários \\
8 & $14 \mathrm{a}$ & $\mathrm{F}$ & Lábios fechados & Bilateral & Ausência de septo pelúcido + nódulos subependimários \\
9 & $2 \mathrm{a}$ & $\mathrm{F}$ & Lábios fechados & Unilateral & Ausência de septo pelúcido + coleção subdural extensa \\
10 & $3 \mathrm{a}$ & $\mathrm{F}$ & Lábios abertos & Bilateral & Ausência de septo pelúcido \\
11 & $9 \mathrm{a}$ & $\mathrm{M}$ & Lábios abertos e fechados & Bilateral & Ausência de septo pelúcido + nódulos subependimários \\
\hline
\end{tabular}

Pac, paciente; M, masculino; F, feminino. 
Tabela 2. Achados clínicos, antecedentes obstétricos e outros achados relevantes nos pacientes com diagnóstico de esquizencefalia.

\begin{tabular}{|c|c|c|c|c|c|}
\hline \multirow[t]{2}{*}{ Pac } & \multicolumn{3}{|c|}{ Clínica } & \multirow[t]{2}{*}{ Antecedentes obstétricos } & \multirow[t]{2}{*}{ Outros } \\
\hline & Epilepsia & Motores & DNPM & & \\
\hline 1 & $\begin{array}{c}\text { Generalizada } \\
\text { tônicoclônica há } 2 \text { meses }\end{array}$ & $\begin{array}{l}\text { Tetraparesia } \\
\text { espástica }\end{array}$ & Atrasado & $\begin{array}{l}\text { Parto normal; nascido } \\
\text { em más condições }\end{array}$ & $\begin{array}{c}\text { Microcefalia }+ \\
\text { estrabismo }\end{array}$ \\
\hline 2 & Ausente & $\begin{array}{c}\text { Tetraparesia } \\
\text { espástica c/ } \\
\text { predomínio à E }\end{array}$ & Atrasado & HAS & Disfagia para sólidos \\
\hline 3 & $\begin{array}{l}\text { Inespecífica desde o } \\
\text { nascimento }\end{array}$ & $\begin{array}{l}\text { Hemiparesia } \\
\text { espástica à D }\end{array}$ & Atrasado & $\begin{array}{c}\text { Sem acompanhamento } \\
\text { pré-natal }\end{array}$ & $\begin{array}{l}\text { Disfagia a sólidos } \\
\text { e líquidos }\end{array}$ \\
\hline 4 & Inespecífica & $\begin{array}{c}\text { Hipertonia } \\
\text { generalizada }\end{array}$ & Atrasado & Sem intercorrências & $\begin{array}{c}\text { Nistagmo }+ \\
\text { estrabismo }+ \\
\text { fontanela anterior } \\
\text { ampla }\end{array}$ \\
\hline 5 & Ausente & $\begin{array}{l}\text { Hemiparesia } \\
\text { espástica à E }\end{array}$ & Atrasado & Sem intercorrências & $\begin{array}{c}\text { Déficit pôndero- } \\
\text { estatural + } \\
\text { artrogripose }\end{array}$ \\
\hline 6 & Ausente & Ausente & Atrasado & $\begin{array}{c}\text { Sem acompanhamento } \\
\text { pré-natal }\end{array}$ & $\begin{array}{c}\text { Cefaléias e vômitos } \\
+ \text { hidrocefalia } \\
\text { obstrutiva }\end{array}$ \\
\hline 7 & Ausente & $\begin{array}{l}\text { Hemiparesia } \\
\text { espástica }\end{array}$ & $\begin{array}{l}\text { Normal ao } \\
\text { diagnóstico }\end{array}$ & $\begin{array}{l}\text { Cianose ao nascimento e } \\
\text { icterícia no } 3 .^{\circ} \text { dia de vida }\end{array}$ & Estrabismo \\
\hline 8 & $\begin{array}{l}\text { Parcial localizada } \\
\text { há } 2 \text { anos }\end{array}$ & Hipertonia global & Atrasado & $\begin{array}{c}\text { Sem acompanhamento } \\
\text { pré-natal }\end{array}$ & $\begin{array}{l}\text { Sinal de Babinski } \\
\text { bilateral }\end{array}$ \\
\hline 9 & Generalizada há 1 ano & $\begin{array}{c}\text { Tetraparesia } \\
\text { espástica }\end{array}$ & Atrasado & Cianose ao nascimento & $\begin{array}{c}\text { Hidrocefalia }+ \\
\text { nistagmo }+ \text { ptose } \\
\text { palpebral bilateral }+ \\
\text { estrabismo }+ \text { atrofia } \\
\text { de papila }\end{array}$ \\
\hline 10 & $\begin{array}{l}\text { Generalizada } \\
\text { tônico-clônica }\end{array}$ & $\begin{array}{l}\text { Tetraparesia } \\
\text { espástica }\end{array}$ & Atrasado & Cianose ao nascimento & Marcha atáxica \\
\hline 11 & Ausente & $\begin{array}{l}\text { Tetraparesia } \\
\text { espástica + } \\
\text { espasmos } \\
\text { musculares }\end{array}$ & Atrasado & $\begin{array}{c}\text { Sem acompanhamento } \\
\text { pré-natal }\end{array}$ & $\begin{array}{l}\text { Disfagia }+ \text { sinal de } \\
\text { Babinski bilateral }\end{array}$ \\
\hline
\end{tabular}

HAS, hipertensão arterial sistêmica; DNPM, desenvolvimento neuropsicomotor; D, direita; E, esquerda; Pac, paciente.

distância variável da fenda, até circunvoluções aberrantes, que convergem para ela. Outras alterações que podem estar associadas são as heterotopias nodulares subependimais, o septo pelúcido incompleto ou ausente e a hipoplasia ou atrofia do tálamo. Os gânglios da base, o cerebelo e o tronco cerebral estão usualmente normais ${ }^{15}$.

A sintomatologia dos pacientes que apresentam esquizencefalia é bastante variada, sendo que a gravidade de alguns sintomas está relacionada à extensão do defeito anatômico e à sua localização no encéfalo ${ }^{6,14-18,}$. Os achados mais frequentes, segundo a literatura, são a epilepsia, o déficit motor e o retardo mental ${ }^{3,9,10}$. Este fato foi confirmado neste estudo, todos os pacientes (exceto o Caso 6) apresentavam algum tipo de déficit motor (hemiparesia, tetraparesia ou hipertonia), 10 pacientes $(90,9 \%)$ apresentavam atraso no DNPM e 6 (54,5\%) epilepsia.

Tipicamente as fendas são encontradas na região peri-silviana ${ }^{1,2,9}$. Há casos de fissuras parassagitais, occipitais e na face orbitária dos lobos frontais ${ }^{6,9,10}$. Fendas bilaterais são mais comuns ${ }^{6}$. Neste estudo, havia 4 casos com fendas unilaterais ( $36 \%$ ) e $7 \mathrm{com}$ fendas bilaterais (64\%).

As esquizencefalias bilaterais associam-se a atrasos mentais severos e importantes déficits motores ${ }^{9}$. 
Tabela 3. Quadro comparativo entre achados deste estudo e os da literatura.

\begin{tabular}{|c|c|c|c|c|}
\hline & Achados do presente estudo & Barkovich JA, Kjos BO & Bird CR, Gilles FH & $\begin{array}{c}\text { Granata T, Battaglia G, } \\
\text { D'Incerti L et al. }\end{array}$ \\
\hline $\mathrm{N}$ & 11 & 20 & 8 & 9 \\
\hline Fendas unilaterais & 4 & 13 & 8 & 6 \\
\hline Fendas bilaterais & 7 & 7 & 0 & 3 \\
\hline Epilepsia & 6 & 16 & 3 & 6 \\
\hline Atraso DNPM & 10 & 13 & 4 & 4 \\
\hline Déficit motor & $\begin{array}{l}3 \text { hemiparesia } \\
7 \text { tetraparesia }\end{array}$ & $\begin{array}{l}10 \text { hemiparesia } \\
5 \text { tetraparesia }\end{array}$ & $\begin{array}{l}7 \text { hemiparesia } \\
1 \text { normal }\end{array}$ & $\begin{array}{l}1 \text { hemiparesia } \\
1 \text { diplegia } \\
2 \text { tetraparesia }\end{array}$ \\
\hline Outros achados & $\begin{array}{c}9 \text { ausência septo pelúcido; } \\
4 \text { nódulos subependimários; } \\
1 \text { microcefalia }\end{array}$ & $\begin{array}{c}3 \text { córtex cerebral } \\
\text { displásico; } \\
2 \text { heterotopia de } \\
\text { substância cinzenta; } \\
1 \text { múltiplas anomalias }\end{array}$ & $\begin{array}{l}2 \text { polimicrogiria; } \\
6 \text { hidrocefalia } \\
\text { mínima; } \\
4 \text { microcéfalos }\end{array}$ & $\begin{array}{c}2 \text { polimicrogiria; } \\
2 \text { heterotopia de substância } \\
\text { cinzenta; } \\
1 \text { ausência de septo pelúcido; } \\
1 \text { ausência parcial de corpo caloso; } \\
1 \text { displasia córtex cerebral; } \\
1 \text { cisto aracnóide }\end{array}$ \\
\hline
\end{tabular}

N, número de pacientes estudados; DNPM, desenvolvimento neuropsicomotor.

Dos 7 pacientes analisados, que possuíam fendas bilaterais, havia atraso no DNPM em $6(85,71 \%)$, tetraparesia em $3(42,85 \%)$ e hemiparesia em 1 $(1,42 \%)$. Estes dados, concordam com o relato de Aniskiewicz et al. ${ }^{18}$, o qual refere que o grau de comprometimento, tanto do desenvolvimento intelectual como do déficit motor, está diretamente relacionado à quantidade de massa encefálica comprometida. Barkovich e Kjos, em estudo publicado em 1992, também encontram dados similares ${ }^{14}$.

Já a presença ou a severidade da epilepsia não estão necessariamente, relacionadas à quantidade de massa encefálica perdida ${ }^{16}$. Todavia, em 4 dos nossos pacientes com lesão bilateral, a epilepsia estava presente $(57,12 \%)$ e era de difícil controle (casos $1,4,8$ e 10$)$.

As fendas unilaterais grandes têm moderado a severo atraso psicomotor. As fendas unilaterais menores, manifestam-se com distúrbios motores contralaterais ou convulsões, porém, as crianças afetadas, em geral, têm o desenvolvimento intelectual nor$\mathrm{mal}^{14}$. Em nosso estudo, 4 pacientes apresentavam fendas unilaterais. Destes, 2 (50\%) apresentavam hemiparesia, $2(50 \%)$ tetraparesia e 1 (25\%) hipertonia e 2 (50\%) pacientes referiam epilepsia (Casos 3 e 9). Estes dados concordam com a literatura. A Tabela 3 visa comparar os achados deste estudo com a literatura pertinente.

A avaliação diagnóstica das esquizencefalias pode ser feita por TC e ressonância magnética (RM) ${ }^{9}$. Ve- rificam-se fendas uni ou bilaterais, que variam de muito estreitas (lábios fechados) até muito largas (Figs 1, 2, 3) . Quatro achados que permitem fazer o



Fig 1. Esquizencefalia de lábios fechados à direita e abertos à esquerda. A fenda direita apresenta-se como uma faixa isodensa à cortical, que se estende da superfície ao ventrículo lateral. Ver também a imagem triangular na parede do ventrículo esquerdo em sua junção com a fenda ("bico"). 


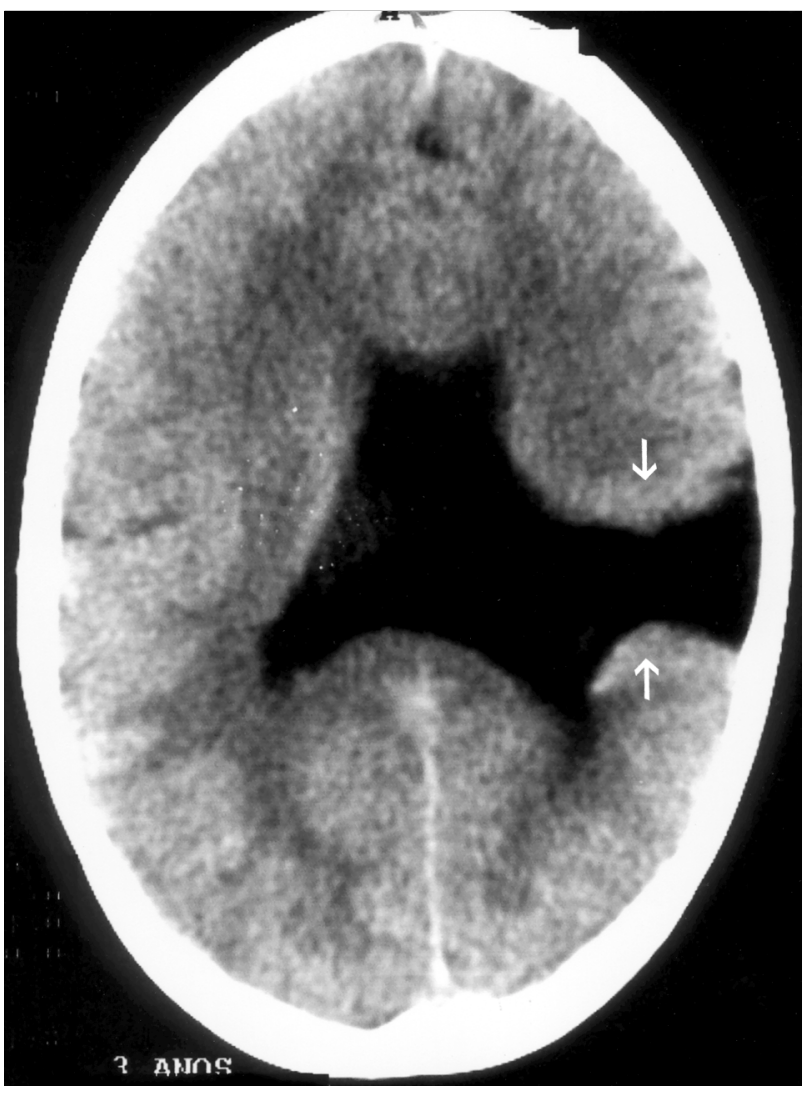

Fig 2. Esquizencefalia unilateral de lábios abertos. Não se identifica o septo pelúcido.

diagnóstico das anomalias do desenvolvimento e diferenciá-las de processos destrutivos nos exames de imagem:

- presença de fendas circundadas por córtex anormal (Figs 1-3);

- o ventrículo tem configuração triangular adjacente à fenda ("bico"), sem dilatação (Fig 1);

- visualiza-se um vaso anômalo entre as superfícies corticais da fenda ${ }^{11}$;

- a ausência de septo pelúcido ${ }^{6,11}$ (Figs 1- 3)

À exceção dos vasos anômalos, nossos pacientes apresentavam os achados radiológicos supramencionados.

O principal diagnóstico diferencial da esquizencefalia é a porencefalia. Nesta, ocorre uma lesão destrutiva do parênquima encefálico no período intrauterino, porém após o término da migração, resultando numa cavidade, que também comunica o sistema ventricular com o espaço sub-aracnóide, porém não se identifica substância cinzenta disgenética nas suas bordas ${ }^{15}$. A diferenciação da esquizencefalia com a porencefalia tem importância no aconselhamento genético. Sabe-se que a anomalia cerebral é 5 a $20 \%$ mais frequente nos casais que tiveram um

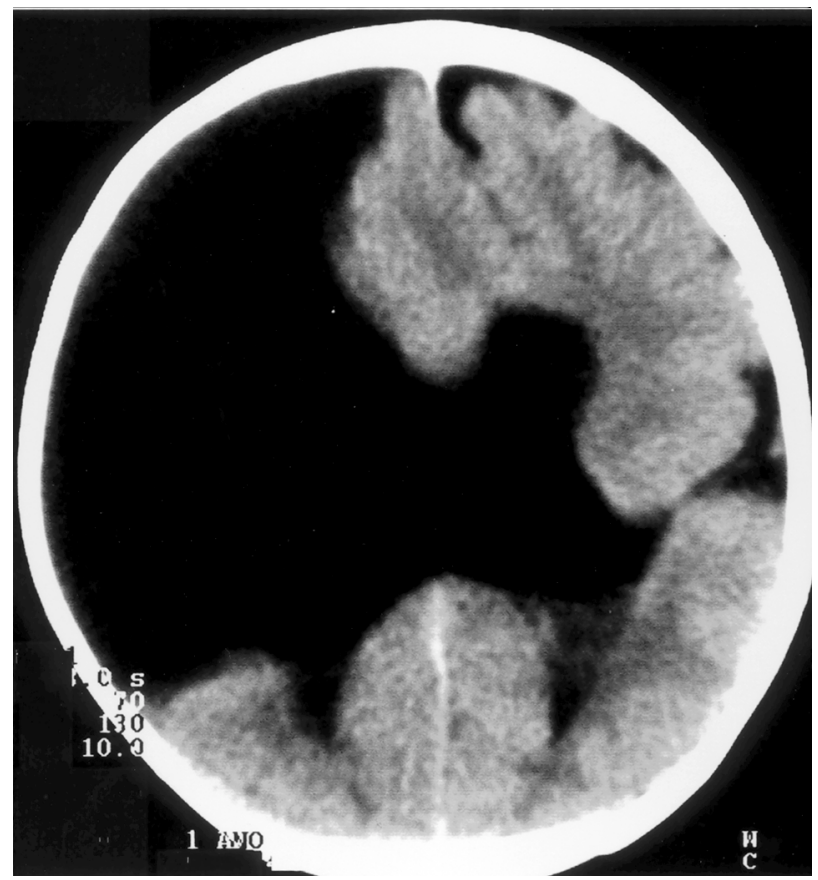

Fig 3. Esquizencefalia bilateral de lábios abertos: há grande redução do parênquima encefálico à direita.

filho com esquizencefalia ${ }^{6,19,20}$. Além disso, um diagnóstico correto permite planejamento terapêutico adequado e específico. Já a expectativa de vida destes pacientes depende basicamente do grau de comprometimento neurológico, haja vista a grande incidência de infecções respiratórias decorrente de aspiração nos pacientes severamente comprometidos ${ }^{15}$.

A RM é o exame de imagem padrão-ouro para o diagnóstico de esquizencefalias, sendo, de forma geral, mais específica e mais sensível que a TC, devido a sua capacidade de melhor diferenciação entre substância cinzenta e branca, permitindo a demonstração multiplanar da anatomia ${ }^{10,21}$ e de outras anomalias associadas, como heterotopias de substância cinzenta, ausência de septo pelúcido e polimicrogirias $^{3}$.

Embora menos sensível, a TC é muito útil pelo seu menor custo, maior disponibilidade e por permitir uma demonstração anatômica suficiente para estabelecer o diagnóstico e o prognóstico, desde que os aspectos típicos da esquizencefalia sejam reconhecidos no exame.

\section{REFERÊNCIAS}

1. Yakovlev PI, Wadsworth RC. Schizencephalies: a study of the congenital clefts in the cerebral mantle. I. Clefts with fused lips. J Neuropathol Exp Neurol 1946;5:116-130.

2. Yakovlev PI, Wadsworth RC. Schizencephalies : a study of the congenital clefts in the cerebral mantle. II. Clefts with hydrocephalus and lips separated. J Neuropathol Exp Neurol 1946;5:169-206. 
3. Barkovich JA, Chuang SH, Norman D. MR of neuronal migration anomalies. AJNR 1988;9:1009-1017.

4. Knapp MS, Valk J. Classification of congenital abnormalities of the CNS. ANJR 1988;9:315-326

5. Sato Y, Kao SC, Smith WL. Radiographic manifestations of anomalies of the brain. Radiol Clin N Am 1991;29:179-193.

6. Miller GM, Stears JC, Guggenheim MA, et al. Schizencephaly : a clinical and CT study. Neurology 1984;34:997-1001.

7. Packard Am, Miller VS, Delgado MR. Schizencephaly: correlations of clinical and radiologic features. Neurology 1997;48:1427-1434.

8. Heschl R. Gehirndefect und Hydrocephalus. Vjschr Prakt Heilk 1859;59-74.

9. Bird CR, Gilles FH. Type I schizencephaly: CT and neuropathologic findings. AJNR 1984;8:451-454.

10. Barkovich JA, Norman D. MR imaging of schizencephaly. AJNR 1988;9:297-302.

11. Zimmerman RA, Bilaniuk LT, Grossmann RI. Computed tomography in migratory disorders of human brain development. Neuroradiology 1983;25:257-263.

12. Richman DP, Stewart RM, Caviness VS Jr. Cerebral microgyria in a 27week fetus : an architectonic and topographic analysis. J Neuropathol Exp Neurol 1974;33:374-384.
13. Volpe JJ. Neurology of the newborn. 2.Ed. Philadelphia: Saunders, 1987.

14. Barkovich AJ, Kjos BO. Schizencephaly: correlation of clinical findings with MR characteristics. AJNR 1992;13:85-94.

15. Ball WS Jr. Pediatric neuroradiology. Philadelphia: Lippincott-Raven 1996:91-174.

16. Granata T, Battaglia G, D'Incerti L, et al. Schizencephaly: neuroradiologic and epileptogenci findings. Epilepsia 1996;37:1185-1193.

17. Volpe JJ. In Schaffer AJ. (ed.) Major problems in clinical pediatrics, Vol XXII. Philadelphia: Saunders, 1981.

18. Aniskiewicz AS, Frumkin NL, Brady DE, Moore JB, Pera A. Magnetic resonance imaging and neurobehavioral correlates in schizencephaly. Arch Neurol 1990;47:911-916.

19. DobynsWB, StrattonRF, Greenberg F. Syndromes with lissencephaly: I. Miller-Dieker and Norman-Roberts syndromes and isolated lissencephaly. Am J Med Genet 1984;18:509-526.

20. Dobyns WB, Kirkpatrick JB, Hittner HM, Roberts RM, Kretzer FL. Syndromes with lissencephaly. II. Walker-Warburg and cerebro-oculomuscular syndromes and a new syndrome with type II lissencephaly. Am J Med Genet 1985;22:157-195.

21. Osborn RE, Byrd SE, Naidich TP, et al. MR imaging of neuronal migrational disorders. AJNR 1988; 9:1101-1106. 